\section{VP191 Peripheral Nerve Field Stimulation For Chronic Low Back Pain}

\section{AUTHORS:}

Marc Rhainds (marc.rhainds@chuq.qc.ca), Brigitte Larocque, Sylvain Bussières, Alice Nourrisat, Martin Coulombe, Léo Cantin, Jean-Francois Canuel, Michel Prudhomme

\section{INTRODUCTION:}

Despite numerous medical, pharmacological and surgical approaches for chronic low back pain (LBP), many patients continue to complain of severe disabling pain. Peripheral nerve field stimulation (PNfS), alone or combined with spinal cord stimulation, is a neuromodulation procedure that have been recently developed and implemented in our hospital. We conducted a Health Technology Assessment (HTA) to determine if PNfS may be considered as a standard of practice in the management of intractable LBP and failed back surgery syndrome (FBSS).

\section{METHODS:}

An interdisciplinary group of experts was involved in the project. A systematic review (SR) was performed in several databases and grey literature to identify clinical practice guidelines, SR and observational studies published through September 2016. A survey was conducted among other chronic pain centers in Canada to document PNfS use in LBP and FBSS treatment.

\section{RESULTS:}

Data on effectiveness and safety of PNfS in chronic LBP treatment were scarce. Short-term results (3-12 months) from small sample and low quality studies suggest that PNfS, alone or combined with spinal cord stimulation, is associated with pain intensity and opioid use reductions. Effects on functional status and quality of life remain undetermined. Most frequent adverse events reported with PNfS devices are lead migrations, discomfort or pain and surgical site infections. No other Canadian pain centers were found to use PNfS in chronic LBP or FBSS.

\section{CONCLUSIONS:}

PNfS is potentially a beneficial treatment option for patients with chronic low back pain or FBSS. However, the value of this innovative treatment remains unknown. Among factors to be clarified are target population (any chronic low back pain or FBSS), use of PNfS alone or combined with spinal cord stimulation, long-term effects, and comparison with conventional medical management. PNfS use in chronic LBP has to be assessed through a rigorous framework before its introduction as a standard medical practice.

\section{VP192 Importance Of Contextual Data In Producing Health Technology Assessment Recommendations}

\section{AUTHORS:}

Thomas Poder (tpoder.chus@ssss.gouv.qc.ca), Christian Bellemare

\section{INTRODUCTION:}

Available data in the scientific literature is not always sufficient to make a clear Health Technology Assessment (HTA) recommendation without any other source of data. Contextual data and local expertize are an important source of data that cannot be ignored in HTA process. Despite a lack of evidence in the scientific literature, a technology can be recommended in a given context. We illustrate this by a case study about biplane angiography for vascular neurointervention.

\section{METHODS:}

A systematic review was conducted. The level of evidence was assessed by the grid of Downs and Black. An analysis of the context in our setting was also conducted. The main outcomes were: radiation doses, clinical complications, procedure times, purchase cost, impact on teaching programs, confidence of clinicians in the technology, quality of care, and volume of activity. A committee constituted of managers, clinical experts, physicians, physicists and HTA experts was 
created to produce a recommendation about biplane technology acquisition.

\section{RESULTS:}

A list of 257 citations was obtained yielding 9 eligible articles for analysis. Despite the lack of evidence found in the literature (median of Downs and Black: 3/32), the biplane system appears to reduce ionizing radiation and medical complications as well as shorten procedure time. Contextual data indicated that biplane system could improve operator's confidence, which could translate into reduced risk, especially for complex procedures. We estimate that a minimum of 50 cases can be done in our context (University hospital center covering a population of 306,322 people) with a higher level of patient safety. In addition, the biplane system can support our institution in advanced procedures teaching program.

\section{CONCLUSIONS:}

Given on the advantages provided by the biplane technology in our setting, the committee has recommended its acquisition. However, this technology should be implemented with a responsibility in collecting outcome data to optimize clinical protocol in doses of ionizing delivered.

\section{VP194 Health Technology Assessment Applied To Nurse Retention And Development: A Sickle Cell Example}

\section{AUTHORS:}

Julia Lavenberg, Matthew Mitchell (mdmitchell@ uphs.upenn.edu), Kendal Williams, Craig Umscheid

\section{INTRODUCTION:}

Health Technology Assessment (HTA) methods are usually applied to the evaluation of drugs, devices, and procedures. We have used HTA to promote evidence-based decision-making on topics relating to staffing and career development for healthcare professionals. Interventions to reduce the stress associated with caring for patients who need repeated hospitalization such as patients with sickle cell disease are thought to improve job satisfaction and nurse retention, but is there scientific evidence to support them?

\section{METHODS:}

We systematically searched Medline, CINAHL, PsycINFO, Cochrane, and Joanna Briggs Institute databases for published studies evaluating interventions targeting healthcare personnel. Searches combined tems for sickle cell disease with terms for job stress, turnover, and other career-related outcomes. We evaluated the quality of individual studies using standardized checklists and constructed evidence tables.

\section{RESULTS:}

We found one randomized trial (RCT) of an education program for nurses and physicians, a pre-post analysis of a communication skills and cultural awareness program, and a case study of a nurse support group. The RCT found that an education program significantly improved participants attitude towards patients but did not measure any outcomes relating to caregiver stress or job satisfaction. The pre-post study found that a communication skills program significantly improved nurses confidence in their ability to communicate with patients. The case study reported that nurses found the support group useful and felt their attitudes were improved, but there was no control group to compare their responses to. The education program was graded as moderate-strength evidence and the other programs had low-strength evidence. There was no meta-analysis or other data synthesis of the results because of the differing interventions and outcome measures.

\section{CONCLUSIONS:}

There have been few quantitative scientific evaluations of the effectiveness of interventions to reduce the stress nurses feel when caring for sickle cell disease patient. The studies that have been published have favorable conclusions towards these interventions, but the strength of evidence is not high. 